\title{
SEPARATELY CONTINUOUS FUNCTIONS: APPROXIMATIONS, EXTENSIONS, AND RESTRICTIONS
}

\section{ZBIGNIEW PIOTROWSKI and ROBERT W. VALLIN}

Received 23 August 2002

\begin{abstract}
A function $f(x, y)$ is separately continuous if at any point the restricted functions $f_{x}(y)$ and $f_{y}(x)$ are continuous as functions of one variable. In this paper, we use several results which have been obtained for other generalized continuities and apply them to functions which are separately continuous.
\end{abstract}

2000 Mathematics Subject Classification: 54C30.

1. Introduction. In this paper, we work with functions $f$ from $\mathbb{R} \times \mathbb{R}$ into $\mathbb{R}$, but note here that many of the definitions and results can be suitably generalized in case if the domain space of $f$ is $\mathbb{R}^{n}$. Cauchy, in 1821 , wrote that a function of several variables which is continuous in each variable separately is continuous as a function of all the variables. This is, of course, false, the first counterexample appearing in 1873 is as follows.

EXAMPLE 1.1. Let $f: \mathbb{R} \times \mathbb{R}$ be defined by

$$
f(x, y)= \begin{cases}\frac{2 x y}{x^{2}+y^{2}}, & (x, y) \neq(0,0) \\ 0, & (x, y)=(0,0)\end{cases}
$$

This function is continuous everywhere except $(0,0)$, where it is continuous along the lines $x=0$ and $y=0$.

The fact that this is continuous when reduced to a one-variable function, but not as a function of two variables, leads us to the following definition.

Definition 1.2. Let $f: \mathbb{R} \times \mathbb{R} \rightarrow \mathbb{R}$. For every fixed $x \in \mathbb{R}$, the function $f_{x}: \mathbb{R} \rightarrow \mathbb{R}$ defined by

$$
f_{x}(y)=f(x, y)
$$

is called an $x$-section of $f$. The $y$-section is similarly defined.

We say $f: \mathbb{R} \times \mathbb{R} \rightarrow \mathbb{R}$ is separately continuous if each $x$-section and each $y$-section is a continuous function. 
So our first example tells us that a function which is separately continuous in both variables is not the same as a continuous function. In this paper, we also work with another generalization of continuous functions called quasicontinuous functions and some variations of that notion. We state their definitions here.

DEFINITION 1.3. Let $f: \mathbb{R} \times \mathbb{R} \rightarrow \mathbb{R}$. Then

(1) $f$ is quasicontinuous at $(x, y)$ if for each $U$ and $V$ open in $\mathbb{R}$ with $(x, y) \in$ $U \times V$ and open set $W \subset \mathbb{R}$, where $f(x, y) \in W$, there is an open set $U^{\prime} \subset U$ and an open set $V^{\prime} \subset V$ such that

$$
f\left(U^{\prime} \times V^{\prime}\right) \subset W
$$

(2) $f$ is quasicontinuous with respect to $x$ (alternatively $y$ ) if we also insist $x \in U^{\prime}\left(y \in V^{\prime}\right)$;

(3) $f$ is symmetrically quasicontinuous if it is quasicontinuous with respect to $x$ and $y$.

The relationships between these various notions are summarized in the following diagram where $C$ represents the continuous functions, SC the separately continuous functions, QC the quasicontinuous functions, SQC the separately quasicontinuous functions, and SymQC the symmetrically quasicontinuous functions:

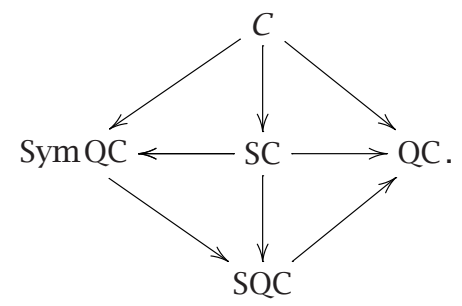

There is an abundance of examples to show that none of these arrows may be reversed.

We note here that a major difference between separately continuous and quasicontinuous functions is the so-called Sierpiński property [7]. The property concerns the ability of a function to be uniquely based on its values on a dense set in the domain.

SIERPIŃSKI PROPERTY. Any real-valued separately continuous function is determined by its values on any dense subset of the domain space. That is, if $f$ and $g$ are separately continuous and agree on a dense set $D$ in the domain space, then $f$ and $g$ agree everywhere.

The following example shows that the Sierpiński property does not hold even for symmetrically quasicontinuous functions. 
EXAMPLE 1.4. Let $f$ and $g$ from $\mathbb{R} \times \mathbb{R}$ into $\mathbb{R}$ be defined by

$$
\begin{aligned}
& f(x, y)= \begin{cases}\sin \left(\frac{1}{x^{2}+y^{2}}\right), & (x, y) \neq(0,0), \\
1, & (x, y)=(0,0),\end{cases} \\
& g(x, y)= \begin{cases}\sin \left(\frac{1}{x^{2}+y^{2}}\right), & (x, y) \neq(0,0), \\
0, & (x, y)=(0,0) .\end{cases}
\end{aligned}
$$

Then $g$ and $f$ are symmetrically quasicontinuous and agree on the dense set $\mathbb{R}^{2} \backslash\{0\}$, but they are not equal.

In this paper, we look at several different types of results for functions having various generalizations of continuity and reformulate them in terms of separately continuous functions. Various examples are also given to show that separate continuity is an important ingredient in the hypotheses.

2. Approximations. Many papers have been written concerning approximating a function as a pointwise limit. Probably the most well-known class of pointwise limits are the Baire-one functions, the functions which are the pointwise limit of continuous functions. In this section, we show that separately continuous functions from $\mathbb{R}^{2}$ to $\mathbb{R}$ are a pointwise limit of what we call planar approximation functions. The main motive behind this section was the following result from [8] concerning a type of almost continuous function. A function $f:[0,1] \rightarrow \mathbb{R}$ is called almost continuous (in the sense of Stallings) if for every open set $U$ containing the graph of $f$, there is a continuous $g:[0,1] \rightarrow \mathbb{R}$ whose graph is contained in $U$.

THEOREM 2.1. Every almost continuous (in the sense of Stallings) function $f:[0,1] \rightarrow \mathbb{R}$ is polygonally almost continuous.

Another way of saying this is, for every open set $U$ containing the graph of $f$, there is in $U$ the graph of a polygonal function $g:[0,1] \rightarrow \mathbb{R}$ whose vertices lie on the graph of $f$. Our way of considering at this is that there is a sequence of polygonal functions converging pointwise to $f$. We wish to redo this result in terms of functions whose domain is the unit square.

Instead of approximations by line segments, we approximate by pieces of a plane. Let $f:[0,1] \times[0,1] \rightarrow \mathbb{R}$. We define $P_{n}(x, y)$, the planar approximations to $f$, as follows: for $n=0$, we start with the unit square and divide it into two triangles by splitting it along the diagonal joining $(1,0)$ and $(0,1)$. So our first triangle has corners $(0,0),(1,0)$, and $(0,1)$ while the second triangle has corners $(1,0),(0,1)$, and $(1,1)$. For each triangle, we find the image of the corner points and, using the triples $(x, y, f(x, y))$, we create a planar region through these triples. Adjoining the two planar regions, we obtain our first planar approximation, $P_{0}(x, y)$. 
At stage $n$, divide the unit square into $2^{n}$ subsquares of side length $1 / 2^{n}$. Then divide each square into two triangles for a total of $2^{n+1}$ triangles. So each triangle has vertices $\left(x_{1}, y_{1}\right),\left(x_{2}, y_{2}\right)$, and $\left(x_{3}, y_{3}\right)$ and we use $\left(x_{i}, y_{i}, f\left(x_{i}\right.\right.$, $\left.\left.y_{i}\right)\right), i=1,2,3$, to create a section of a plane in $\mathbb{R}^{3}$. Joining these sections together gives $P_{n}(x, y)$, where for a given $(x, y), P_{n}(x, y)$ is the $z$-value of the plane section above that point. We note here (for later use) that the collection of all the corner points of the triangles used in the approximations is dense in the unit square and will be denoted by $T$.

For some $f$ we cannot recover the function using these $P_{n}$. By this we mean $P_{n}(x, y) \rightarrow f(x, y)$ for some point $(x, y)$. We now show that separately continuous functions are planar approximable. That is, if $f(x, y)$ is separately continuous, then the planar approximation of $f$ converges pointwise to $f$.

THEOREM 2.2. If $f(x, y)$ is separately continuous, then $P_{n}(x, y)$ converges pointwise to $f(x, y)$.

Proof. First, if $(x, y)$ is a corner point of a triangle, the conclusion is obvious.

Second, if $(x, y)$ is a part of a horizontal or vertical boundary for a triangle (without loss of generality, assume that $(x, y)$ is part of a horizontal boundary), but not a corner point, then $(x, y)$ is a bilateral limit of corner points $\left(x_{n}, y_{n}\right)_{r}$ and $\left(x_{n}, y_{n}\right)_{l}$ where $r$ and $l$ refer to left and right sides, respectively, of the $n$th triangle containing $(x, y)$ in its boundary. Since $f$ is separately continuous,

$$
P_{n}\left(x_{n}, y_{n}\right)=f\left(x_{n}, y_{n}\right) \rightarrow f(x, y)
$$

for both the left and right sides. This result coupled with the fact that the boundary of the pieces of the planar approximation are found using linear interpolation between the corners leads us to

$$
P_{n}(x, y) \longrightarrow f(x, y)
$$

Finally, at any other point $(x, y)$ in the unit square, the denseness of the horizontal and vertical boundaries of the triangles along with the same combination of separate continuity of $f$ and linear interpolation in the approximating give us $P_{n}(x, y)$ wich converges to $f(x, y)$.

In order to recover the original function, it is not necessary that $f$ be separately continuous. The following example shows this.

EXAMPLE 2.3. Let $f(x, y)=\chi_{\{(0,0)\}}$, the characteristic function of $(0,0)$. Because the origin is one of the corners for a triangle, the pointwise limit of the planar approximations gives us back the original $f$. This function is not separately continuous.

Our next concern is about the set $\mathscr{P}$ of functions which are the pointwise limit of these $P_{n}$. The previous example shows that $\mathscr{P}$ contains more than just the 
separately continuous functions. The following examples show that although some Baire-one functions and symmetrically quasicontinuous functions are in $\mathscr{P}$, these classes are not contained in $\mathscr{P}$.

EXAMPLE 2.4. Let $a$ be a point not in $T$, then $\chi_{\{a\}}$ (which is obviously Baireone) is not planar approximable.

EXAMPLE 2.5. Pick $\left(x_{0}, y_{0}\right)$ so that $\left(x_{0}, y_{0}\right)$ is not a point on the boundary of any triangle. So there exists a chain of triangles

$$
T_{1} \supset T_{2} \supset T_{3} \supset \cdots
$$

from our development of $T$ with $\left(x_{0}, y_{0}\right) \in T_{i}$ for all $i$. Define $f:[0,1] \times[0,1] \rightarrow$ $\mathbb{R}$ by

$$
f\left(x_{0}, y_{0}\right)=0,
$$

$f \equiv 1$ on the boundary of $T_{i}$ if $i$ is even,

$f \equiv 0 \quad$ on the boundary of $T_{i}$ if $i$ is odd,

and between the triangles everything is connected continuously. Then $f$ is symmetrically quasicontinuous, but the planar approximations at $\left(x_{0}, y_{0}\right)$ jump between 0 and 1 . Thus, $f$ is not planar approximable.

So we know that the separately continuous functions are proper subsets of $\mathscr{P}$ and that $\mathscr{P}$ is a proper subset of the Baire-one function. This gives the following open question.

Problem 2.6. Does there exist a complete description of the functions in $\mathscr{P} ?$

3. Restrictions. In 1922, Blumberg proved the following theorem [1].

THEOREM 3.1. Let $f:[0,1] \rightarrow \mathbb{R}$ be an arbitrary function, then there exists a set $D$, dense in $[0,1]$, such that the restriction of $f$ to $D,\left.f\right|_{D}$, is continuous.

Since then, many "Blumberg type" theorems have been produced. These all have the form if $X$ is a certain type of space and $f: X \rightarrow \mathbb{R}$, then there is a type of dense set $X_{0} \subseteq X$ such that the restriction of $f$ to $X_{0}$ is some type of generalized continuity. A specific example, which is taken from [2], illustrating how "large" the set $X_{0}$ can be, is given in our Theorem 3.2 below. A function $f$ is pointwise discontinuous on $X(f \in \operatorname{PWD}(X))$ if the set of continuity points is dense in $X$.

THEOREM 3.2. If $X$ is a complete metric space dense in itself, then for every $f: X \rightarrow \mathbb{R}$, there exists a c-dense $X_{0} \subset X$ such that $\left.f\right|_{X_{0}} \in \operatorname{PWD}\left(X_{0}\right)$. 
Our "Blumberg type" theorem also tightens the dense set by making it a $c$-dense set and the conclusion is changed to the restricted function being separately continuous.

THEOREM 3.3. For every $f: \mathbb{R}^{2} \rightarrow \mathbb{R}$, there exists a $c$-dense set $D \subset \mathbb{R}^{2}$ such that $\left.f\right|_{D}$ is separately continuous.

PROoF. This can be shown by using either [6] or [3]. The former contains a construction of a $c$-dense set where every horizontal and vertical line intersects at most one point. The latter refers to modifying a result by Mazurkiewicz [4] so that, for any positive integer $n \geq 2$, there is a set in $\mathbb{R}^{2}$ which meets every line in exactly $n$ points. In either case, there is a $c$-dense subset $D$ in the plane. For any $(a, b) \in D$, the horizontal and vertical lines $x=a$ and $y=b$ intersect $D$ at finitely many points. So for $\varepsilon$ small enough, the only points in $(a \pm \varepsilon, b)$ and $(a, b \pm \varepsilon)$ intersected with $D$ will be $(a, b)$. Thus, $\left.f\right|_{D}$ will be separately continuous at $(a, b)$.

4. Extensions. The results for this section have to do with extending a separately continuous function defined on a subset of the plane. Our work is based on the following theorem from [5].

THEOREM 4.1. Let $H \subset[0,1]$ and let $f: H \rightarrow \mathbb{R}$ be continuous and bounded on $H$. Then there exists $h:[0,1] \rightarrow \mathbb{R}$ such that

(1) $h$ is quasicontinuous on $[0,1]$,

(2) $f=h$ on $H$,

(3) $H \subset \mathscr{b}(h)$ where $\mathscr{b}(h)$ is the set of points in $[0,1]$ at which $h$ is continuous.

We begin by changing the domain from the unit interval into the unit square and then show that we can relax the condition on $f$ to a separately continuous function. We then use extra conditions to achieve some corollaries.

THEOREM 4.2. Let $H \subset[0,1] \times[0,1]$ and let $f: H \rightarrow \mathbb{R}$ be separately continuous and bounded on $H$. Then there exists $h:[0,1] \times[0,1] \rightarrow \mathbb{R}$ such that

(1) $h$ is quasicontinuous on $[0,1] \times[0,1]$,

(2) $f=h$ on $H$,

(3) $H \subset \mathscr{C}(h)$ where $\mathscr{C}(h)$ is the set of points interior to $[0,1] \times[0,1]$ at which $h$ is separately continuous.

Proof. All we really need to do is to illustrate how $f(x, y)$ is to be defined for $(x, y)$ on the boundary of $H$ where there are horizontal and/or vertical lines in $H$ approaching the point, and then how to extend it to any points in $[0,1] \times[0,1] \backslash \bar{H}$. It will then be obvious that properties (1), (2), and (3) are true. From the separate continuity of $f$ we should approach a point on the boundary of $H$ from either a horizontal or vertical direction. What we really need to determine is which direction we choose. 
For every $(x, y)$ where $f$ is defined on both the horizontal and vertical line through $(x, y)$, let

$$
f(x, y)=\liminf _{\substack{t \rightarrow y \\(x, t) \in H}} f(x, t) .
$$

For every $(x, y)$ where $f$ is defined on the vertical line through $(x, y)$, but not the horizontal line through the point, then

$$
f(x, y)=\liminf _{\substack{t \rightarrow y \\(x, t) \in H}} f(x, t) .
$$

Finally, for every $(x, y)$ in the boundary of $H$ to which $f$ has not been extended we define $f$ on the horizontal line through $(x, y)$, but not on the vertical line through the point, then

$$
f(x, y)=\liminf _{\substack{s \rightarrow x \\(s, y) \in H}} f(s, y) .
$$

Now, $f$ is defined on $\bar{H}$. If $[0,1] \times[0,1] \backslash \bar{H}$ is nonempty, we can use continuous $x$-sections to finish defining $f$.

EXAMPLE 4.3. In general, we cannot replace Theorem 4.2(1) with " $h$ is symmetrically quasicontinuous on $[0,1] \times[0,1]$."

Proof. Let $H=H_{1} \cup H_{2} \cup H_{3} \cup H_{4}$, where

$$
\begin{array}{ll}
H_{1}=[0,1 / 2) \times[0,1 / 2), & H_{3}=(1 / 2,1] \times[0,1 / 2) \\
H_{2}=[0,1 / 2) \times(1 / 2,1], & H_{4}=(1 / 2,1] \times(1 / 2,1] .
\end{array}
$$

Define $f$ to be 0 on $H_{1}$ and $H_{4}$ while $f$ is 1 on $H_{2}$ and $H_{3}$. It is impossible to extend $f$ to the point $(1 / 2,1 / 2)$ and have it be symmetrically quasicontinuous there.

COROLLARY 4.4. If $H=\cup H_{i}$ where the $\overline{H_{i}}$ are pairwise disjoint, then the extension of $f$ is symmetrically quasicontinuous.

Proof. Because the $\overline{H_{i}}$ are pairwise disjoint, the set $[0,1] \times[0,1] \backslash \cup \overline{H_{i}}$ is an open set and then $h$ can be extended to be continuous on this open set.

Any function which is separately continuous must also be a Baire-one function. The proof of this is due to Lebesgue and is quite elegant. From the Baireone property we obtain the following corollary.

COROLLARY 4.5. If $H=\cup H_{i}$ where the $\overline{H_{i}}$ are pairwise disjoint, then since $f$ is Baire-one the extension is Baire-one.

Proof. This is an immediate consequence of $h$ being continuous on $[0,1] \times$ $[0,1] \backslash \cup \overline{H_{i}}$ and $f$ being Baire-one on $H$. 
5. Linear, not separate, continuity. Related to the separately continuous functions are the linearly continuous functions. A function $f$ is linearly continuous at $(x, y)$ if it is continuous with respect to every line $l$ passing through the point. An early example of a function which is linearly continuous, but not continuous, at the origin was given by W. H. Young and G. C. Young in [9]. We repeat their example.

EXAMPLE 5.1. We will define the function $f: \mathbb{R}^{2} \rightarrow \mathbb{R}$ for the first quadrant only. The other quadrants will then be determined by reflection about the axes. Let $P$ represent the parabola $y=x^{2}$. On both the $x$-axis and $y$-axis, define $f$ to be zero. Between the $y$-axis and the graph of $P$, let $f(x, y)=x^{2} / y$. Between the graph of $P$ and the $x$-axis, let $f(x, y)=y / x^{2}$. Finally, on the parabola itself (except at the origin, where $f$ is zero), set $f(x, y)=1$. It is obvious that $f$ is continuous at every point except the origin. However, for any line $y=m x$ in the first quadrant, we eventually have

$$
f(x, y)=\frac{x^{2}}{y}=\frac{x}{m}
$$

which is continuous at the origin.

We note that W. H. Young and G. C. Young did more than just giving this one example. They took this result and created several more examples, culminating in a function which is linearly continuous, but is discontinuous at uncountably dense many points.

Our result is another one about extensions in the flavor of [5]. This time $f$ begins as linearly continuous and is linearly continuous in the conclusion.

THEOREM 5.2. Let $f: H \subset[0,1] \times[0,1] \rightarrow \mathbb{R}$ be a bounded, linearly continuous function. If $H=\cup_{i=1}^{n} H_{i}$, where $\overline{H_{i}}$ are pairwise disjoint, then there exists an extension $h:[0,1] \times[0,1] \rightarrow \mathbb{R}$ such that $h$ is linearly continuous.

PROoF. Since there are only finitely many $H_{i}$, we can achieve linearly continuity after extending $f$ to each boundary of $H_{i}$. For an arbitrary point $(x, y)$ on the boundary of $H_{i}$ we can define $f(x, y)$ to be

$$
\lim _{\substack{(s, t) \rightarrow(x, y) \\(s, t) \in l}} f(s, t)
$$

where $l$ is any line segment in the interior of $H_{i}$ with $(x, y)$ as an endpoint.

The next example shows that without additional assumptions, it is necessary to have only finitely many $H_{i}$ in order to achieve linear continuity.

EXAMPLE 5.3. This will not necessarily work if $H=\cup_{i=1}^{\infty} H_{i}$. 
Proof. Define $H_{i}$ as $\left[2^{-2 i-2}, 2^{-2 i-1}\right] \times[0,1]$ and define $f$ on $H_{i}$ to be 1 if $i$ is odd and 0 if $i$ is even. There is no way to define $f(0,0)$ so that it is linearly continuous at the origin with respect to the line $y=0$.

THEOREM 5.4. If, instead of having finitely many $H_{i}$, we have $H=\cup_{i=1}^{\infty} H_{i}$, $\overline{H_{i}}$ are pairwise disjoint, and no line intersects infinitely many $H=\overline{H_{i}}$, then we can make the function $h$ to be linearly continuous.

Proof. This holds since for a small enough neighborhood of the point $(x, y)$ in the boundary of $H_{i}$ only finitely many $H_{j}, j \neq i$, can be contained.

We conclude by noting that our earlier section on restrictions can also be applied to linearly continuous functions. This is because Mauldin's result [3] concerns a $c$-dense set which meets any line in at most $n$ places.

ACKNOWLEDGMENTS. This paper was written while the second author was on sabbatical at Youngstown State University. This work was supported by a Research Professorship grant and a University Research Council Grant Award from Youngstown State University to Dr. Zbigniew Piotrowski.

\section{REFERENCES}

[1] H. Blumberg, New properties of real functions, Trans. Amer. Math. Soc. 24 (1922), 113-128.

[2] A. Katafiasz and T. Natkaniec, A new variant of Blumberg's theorem, Real Anal. Exchange 22 (1996/1997), no. 2, 806-813.

[3] R. D. Mauldin, On sets which meet each line in exactly two points, Bull. London Math. Soc. 30 (1998), no. 4, 397-403.

[4] S. Mazurkiewicz, Sur un ensemble plan qui a avec chaque droite deux et seulement deux points communs, C.R. Varsovic 7 (1941), 382-384 (French).

[5] C. J. Neugebauer, Blumberg sets and quasi-continuity, Math. Z. 79 (1962), 451-455.

[6] Z. Piotrowski and E. J. Wingler, On Sierpinski's theorem on the determination of separately continuous functions, Questions Answers Gen. Topology 15 (1997), no. 1, 15-19.

[7] W. Sierpiński, Sur une propriété de fonctions de deux variables réelles, continues par rapport à chacune de variables, Publ. Math. Univ. Belgrade 1 (1932), 125-128 (French).

[8] P. Szuca, Every almost continuous function is polygonally almost continuous, Real Anal. Exchange 25 (1999/2000), no. 2, 691-694.

[9] W. H. Young and G. C. Young, Discontinuous functions continuous with respect to every straight line, Quart. J. of Math. Oxford (Series I) 41 (1910), 87-93.

Zbigniew Piotrowski: Department of Mathematics and Statistics, Youngstown State University, Youngstown, OH 44555, USA

E-mail address: zpiotr@math.ysu.edu

Robert W. Vallin: Department of Mathematics, Slippery Rock University of Pennsylvania, Slippery Rock, PA 16057, USA

E-mail address: robert.va11in@sru.edu 


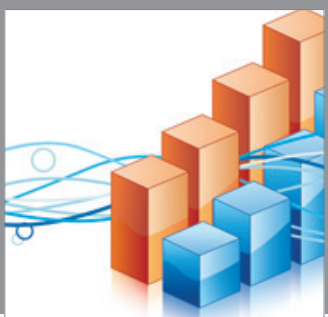

Advances in

Operations Research

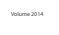

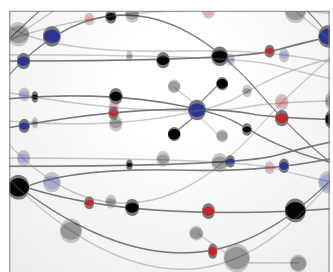

\section{The Scientific} World Journal
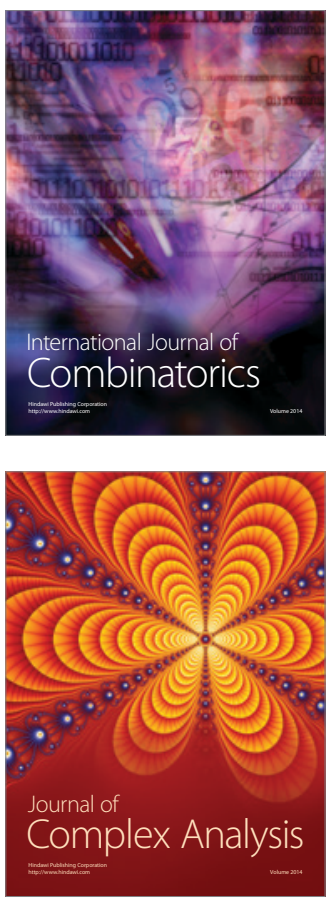

International Journal of

Mathematics and

Mathematical

Sciences
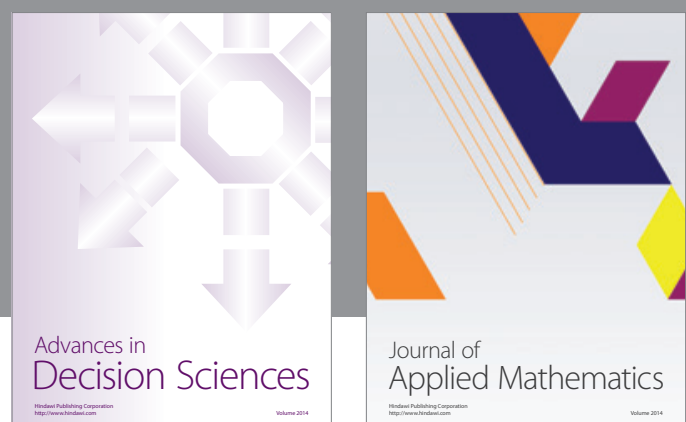

Journal of

Applied Mathematics
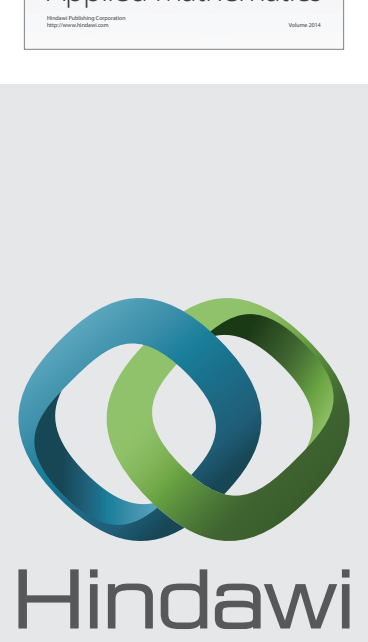

Submit your manuscripts at http://www.hindawi.com
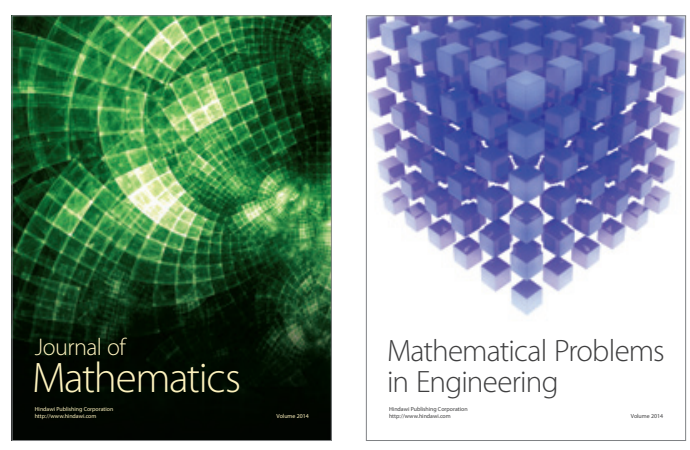

Mathematical Problems in Engineering
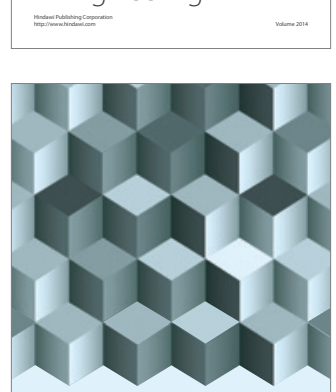

Journal of

Function Spaces
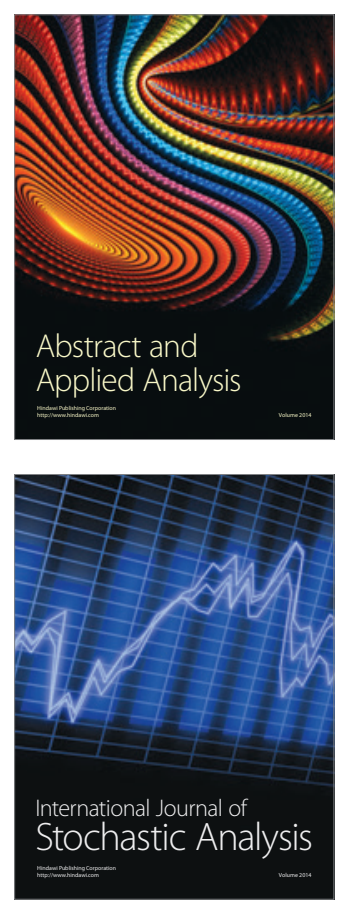

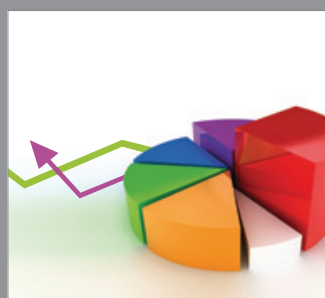

ournal of

Probability and Statistics

Promensencen
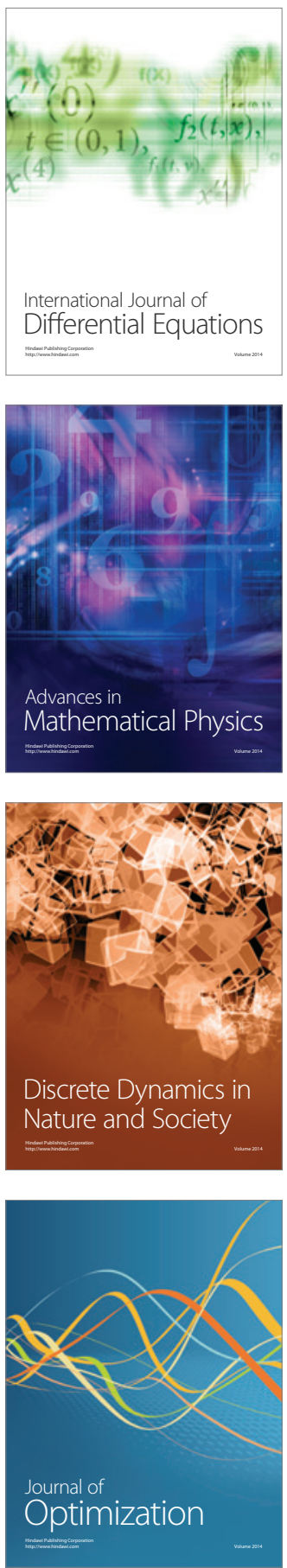